\title{
Regulation and expression of a renin-angiotensin system in human pancreas and pancreatic endocrine tumours
}

\author{
King Yin Lam and Po Sing Leung ${ }^{1}$ \\ Department of Pathology, School of Medicine, James Cook University, Queensland, Australia and ${ }^{1}$ Department of Physiology, Faculty of Medicine, \\ The Chinese University of Hong Kong, Shatin, Hong Kong \\ (Correspondence should be addressed to P S Leung; Email: psleung@cuhk.edu.hk)
}

\begin{abstract}
Objective: Evidence exists for the presence of a renin-angiotensin system (RAS) in the pancreas. The aims of this study were to prove the presence of an intrinsic RAS in the human pancreas and to analyse the role of such an RAS in pancreatic endocrine tumours (PETs).

Methods: Gene expression of key RAS components (angiotensinogen and angiotensin II receptors, namely $\mathrm{AT}_{1}$ and $\mathrm{AT}_{2}$ ) was investigated in human pancreas and in PETs by semi-quantitative RTPCR and immunohistochemistry.

Results: Expression of mRNAs of RAS components was found in human pancreas and in PETs. Data from semi-quantitative RT-PCR analysis demonstrated an increase in the mRNA expression of angiotensinogen and $\mathrm{AT}_{2}$ receptor in PETs when compared with that in normal pancreas. By immunohistochemistry, angiotensinogen protein was predominantly localized in the pancreatic islets while $\mathrm{AT}_{1}$ receptor protein was in the pancreatic ducts.

Conclusions: The data support the notion of the existence of an intrinsic RAS in the human pancreas. It also indicates, for the first time, that such a local pancreatic RAS is subject to regulation by PETs and its significant change may have pathophysiological relevance in patients with PETs.
\end{abstract}

European Journal of Endocrinology 146 567-572

\section{Introduction}

The circulating renin-angiotensin system (RAS) is well known to play important roles in the nervous, cardiovascular and renal systems. The principal hormone of the RAS, angiotensin II, is involved in the regulation of blood pressure, aldosterone secretion and electrolytes as well as fluid homeostasis (1-3). Angiotensin II is spliced from its liver-derived precursor angiotensinogen by the action of an enzyme, renin, secreted by the juxtaglomerular cells in the kidney (4). The hormone acts via the interaction with its specific angiotensin receptors such as $\mathrm{AT}_{1}$ and $\mathrm{AT}_{2}$ (5). In addition to the circulating RAS, major components of the RAS have been shown in numerous tissues/organs of the body like the brain, heart, kidneys, adrenals and gonads (6). These locally generated angiotensins may add to, or differ from, the actions of the circulating RAS through a paracrine/autocrine pathway. Of great interest is the recent evidence for the presence of different components of an RAS in the pancreas of the rat, mouse and dog (7-9). Such an intrinsic, angiotensingenerating RAS has a potential role in finely regulating exocrine and/or endocrine functions of the pancreas (10). In the human pancreas, the expression of an
RAS has only been demonstrated in autopsy materials by Tahmasebi et al. (11). Thus, the role of an RAS in the human pancreas, with particular reference to some pancreatic diseases such as pancreatic endocrine tumour (PET), is largely undefined.

The pancreatic islet is the classical endocrine unit in the pancreas. PETs are endocrine tumours arising from cells in the pancreatic islets (12). They account for slightly more than $10 \%$ of primary pancreatic tumours. This group of tumours is classified according to the clinical and biochemical evidence of hormones they produce. For instance, they can be named as insulinoma, glucagonoma, gastrinoma and so on (12). Among them, insulinoma is the most commonly encountered PET in clinical diagnosis. The tumours present an important challenge to the endocrinologist because of their hormonal manifestations, uncertain malignancy and potential lethality (13). Some interactions may exist among the classical endocrine unit in the pancreas, islet cells and the newly recognized RAS. However, the involvement of a pancreatic RAS in patients with PETs has never been reported in the English language literature.

In this study, we analysed the regulation and localization of the different components of the RAS in normal 
human pancreas. In addition, we examined, for the first time, the association between pancreatic RAS and its clinical implications for PETs. We employed biopsy materials for the present study in order to avoid the autolysis that may be encountered in autopsy specimens.

\section{Materials and methods}

\section{Preparation of tissues from PETs}

Fresh tissues were collected from five patients who underwent resection for insulinomas. In each of these patients, non-tumour pancreatic tissues were also sampled at the Department of Surgery at the University of Hong Kong. Informed consent was obtained from each patient and human ethics approval was sought from the Clinical Research Ethics Committee. These freshly collected specimens were snap frozen in liquid nitrogen and stored at $-70^{\circ} \mathrm{C}$. The other portions of the PETs were fixed in formalin and embedded in paraffin. In addition, formalin-fixed paraffin-embedded blocks of non-tumour pancreases were retrieved from five patients who underwent resection for diseases not related to the pancreas. Haematoxylin and eosinstained sections from all the frozen and paraffinembedded tissues were prepared for the confirmation of the diagnosis of PET and the presence of islets in the non-tumour pancreas. The PETs were proven to be insulin-secreting by both elevated serum insulin levels and tissue expression of insulin (12).

\section{Semi-quantitative RT-PCR analysis of RAS expression}

Total RNA of the frozen samples (normal and tumour pancreases) was isolated according to the acid guanidinium thiocyanate-phenol-chloroform protocol (14). Briefly, tissues were homogenized in $4 \mathrm{~mol} / \mathrm{l}$ guanidinium thiocyanate solution and repeatedly extracted with water-saturated phenol. After extraction with chloroform, RNA was precipitated with isopropanol. The resultant pellet was finally resuspended in water treated with diethylpyrocarbonate. The total RNA was separated by gel electrophoresis and quantified by spectrophotometry. The extracted RNA was then used for first-strand cDNA synthesis using Superscript II transcriptase (GIBCO-BRL, Grand Island, NY, USA) in a final volume of $20 \mu \mathrm{l}$. After incubation at $42{ }^{\circ} \mathrm{C}$ for $1 \mathrm{~h}$, the reaction mixture was treated with RNase $\mathrm{H}$ before proceeding to $\mathrm{PCR}$ analysis. All the RNAs were free of DNA contamination by RT-PCR without addition of reverse transcriptase.

Two preliminary experiments were performed before semi-quantitative RT-PCRs of RAS component genes were analysed as described previously $(15,16)$. To validate the linearity of PCR product accumulation with increasing number of PCR cycles, aliquots containing fixed amounts of cDNA mixture were subjected to amplification for several different PCR cycles, from 20 to 40 cycles. All samples were analysed for both RAS genes and $\beta$-actin gene in the logarithmic phase of the amplification reactions. Fixed amounts of cDNA and optimal PCR cycles were directly employed for individual PCR amplification of human RAS component genes (angiotensinogen, $\mathrm{AT}_{1}$ and $\mathrm{AT}_{2}$ receptors) and human $\beta$-actin genes as employed previously (17). PCR reactions were carried out in a volume of $50 \mu \mathrm{l}$ containing the corresponding sense and antisense primer sequences using the PCR Reagent System (GIBCO-BRL). The PCR conditions were 26-28 cycles of denaturing, at $94^{\circ} \mathrm{C}$ for $1 \mathrm{~min}$, annealing, at $62{ }^{\circ} \mathrm{C}$ for $1 \mathrm{~min}$, and elongating at $72^{\circ} \mathrm{C}$ for $2 \mathrm{~min}$. All PCR products were separated on $2 \%$ agarose gel electrophoresis. The amplified DNA bands were detected using ethidium bromide staining and quantified with an image analyser (Image Quant; Molecular Dynamics, Sunnyvale, CA, USA).

\section{Statistical analysis}

Results of the RT-PCRs are expressed as means \pm S.E.M. ( $n=5$ for each group). The mRNA expression of the various components of the RAS in normal pancreases and PETs was normalized as a percentage of relative expression of RAS components to that of $\beta$-actin. Comparison of differences between individual mean values were made using Student's $t$-test. Values of $P<0.05$ vs respective control groups were considered statistically significant.

\section{Immunohistochemical localization of RAS components}

Immunohistochemical study was conducted on $5 \mu \mathrm{m}$ thick paraffin-embedded sections from normal pancreases and PETs by the avidin-biotin-peroxidase complex method. They were de-paraffinized with xylene and rehydrated through graded concentrations of alcohol. The sections were then treated with microwave irradiation at $95^{\circ} \mathrm{C}$ in $10 \mathrm{mmol} / \mathrm{l}$ citrate buffer for $15 \mathrm{~min}$. They were washed in water, rinsed with Tris-buffered saline (TBS) and treated with 3\% hydrogen peroxide in methanol for $10 \mathrm{~min}$ at room temperature to block the intrinsic peroxidase activity. The sections were again washed with water and rinsed with TBS. Ten per cent normal serum was then added at room temperature for $10 \mathrm{~min}$. Primary antibodies were added to the sections either with anti-angiotensinogen serum diluted at 1:400 as described previously $(7,18)$ or with anti-AT ${ }_{1}$ receptor serum (Santa Cruz Biotech Inc., Santa Cruz, CA, USA) diluted at 1:200 as employed previously (16). The sections were incubated in a moist chamber overnight at $4{ }^{\circ} \mathrm{C}$. The slides were again washed three times in TBS for $3 \mathrm{~min}$. Biotinylated IgG and pre-incubated (30 min at room temperature) avidin-biotin complex (1:100) 
(Amersham International, Amersham, Bucks, UK) were added for $30 \mathrm{~min}$ at $37^{\circ} \mathrm{C}$. They were washed in TBS followed by the development in freshly prepared 3,3'-diaminobenzidine/hydrogen peroxide solution for $10 \mathrm{~min}$ at room temperature. The sections were then washed in water, counter-stained with Mayer's haematoxylin for $1 \mathrm{~min}$ at room temperature, dehydrated, cleaned and mounted. Brown cytoplasmic staining was regarded as a positive signal. For negative control experiments, the specific angiotensinogen and $\mathrm{AT}_{1}$ receptor antibodies were preadsorbed with excesses of their peptide antigens.

\section{Results}

\section{Expression of RAS components in normal pancreas and PETs}

The mRNA expression of angiotensinogen was noted in normal pancreas and PETs (Fig. 1). The RT-PCR
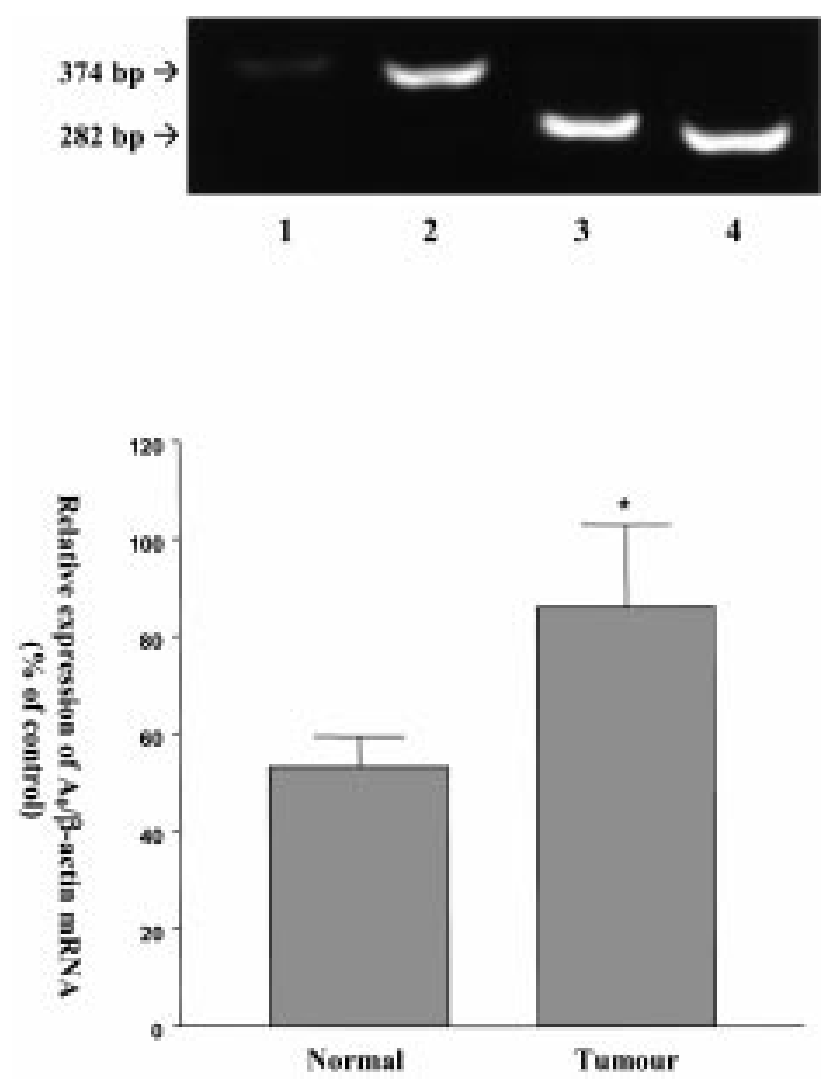

Figure $1 \mathrm{RT}-\mathrm{PCR}$ analysis of mRNA expression for angiotensinogen in PETs. Lane 1, angiotensinogen expression in normal pancreas; lane 2, angiotensinogen expression in a PET; lane 3 , $\beta$-actin expression in control; lane $4, \beta$-actin expression in a PET. The arrows indicate the expected size of amplified products from angiotensinogen ( $374 \mathrm{bp}$ ) and $\beta$-actin ( $282 \mathrm{bp})$. The relative expression is indicated by angiotensinogen $/ \beta$-actin mRNA (\% of control) in normal and in PET pancreas. Data are means \pm S.E.M. ( $n=5$ PETs/group). ${ }^{*} P<0.05$. product was $374 \mathrm{bp}$, as shown in a representative image of gel analysis. The relative expression of mRNAs for angiotensinogen to $\beta$-actin in PETs was increased by approximately 1.5 -fold when compared with those in normal pancreas. The expression of $\mathrm{AT}_{1}$ receptor mRNA was found in normal pancreas and PETs (Fig. 2). The RT-PCR product was $340 \mathrm{bp}$. The relative expression of $\mathrm{AT}_{1}$ receptor in PETs was downregulated to approximately two-thirds when compared with those in normal pancreas. On the other hand, the expression of $\mathrm{AT}_{2}$ receptor mRNA was also detected in normal pancreas and PETs (Fig. 3). The RT-PCR product was $406 \mathrm{bp}$. The relative expression of mRNAs for $\mathrm{AT}_{2}$ receptor was increased by 1.3 -fold when compared with those in normal pancreas, as demonstrated by image analysis.
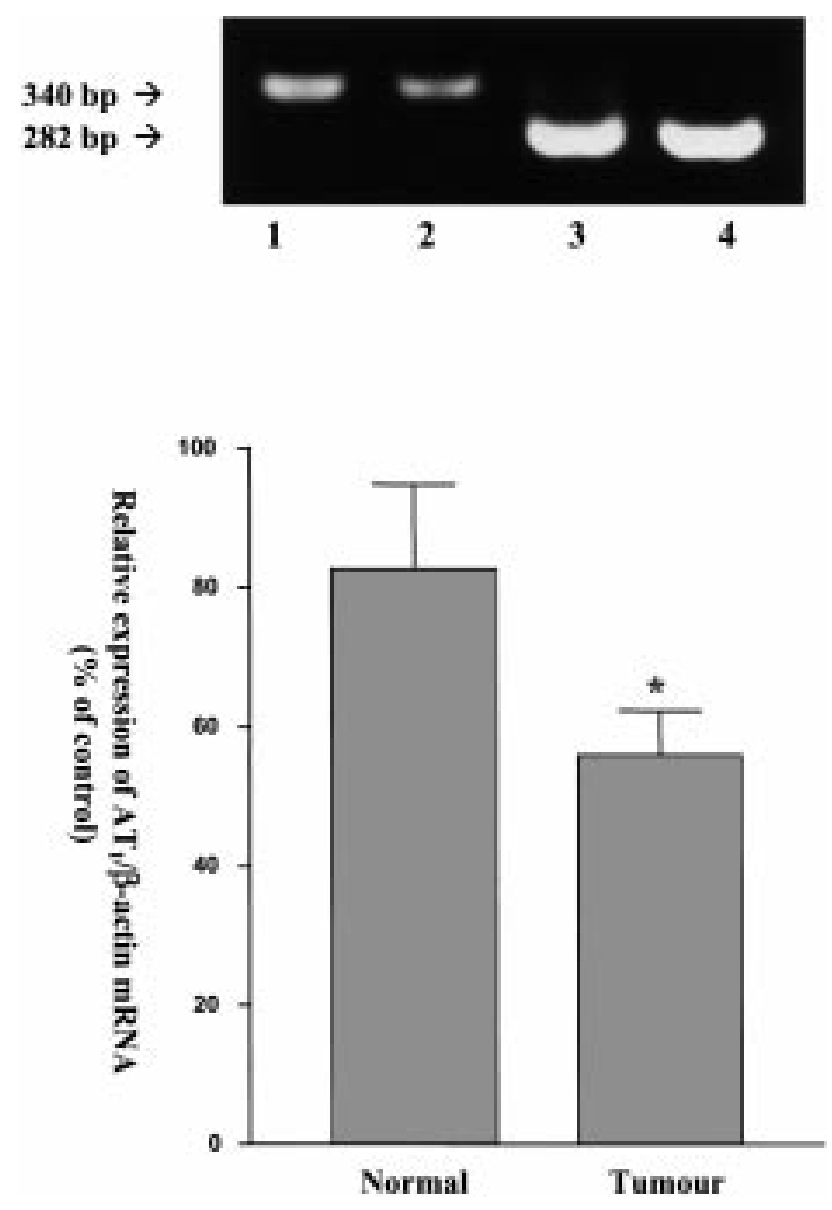

Figure 2 RT-PCR analysis of mRNA expression for $A_{1} T_{1}$ receptor in PETs. Lane 1, $\mathrm{AT}_{1}$ receptor expression in normal pancreas; lane $2, \mathrm{AT}_{1}$ receptor expression in a PET; lane 3 , $\beta$-actin expression in the normal pancreas; lane $4, \beta$-actin expression in a PET. The arrows indicate the expected size of amplified products from $\mathrm{AT}_{1}$ receptor $(340 \mathrm{bp})$ and $\beta$-actin $(282 \mathrm{bp})$. The relative expression is indicated by $A T_{1}$ receptor $/ \beta$-actin mRNA (\% of control) in normal and in PET pancreas. Data are means \pm S.E.M. ( $n=5$ PETs/group). * $P<0.05$. 

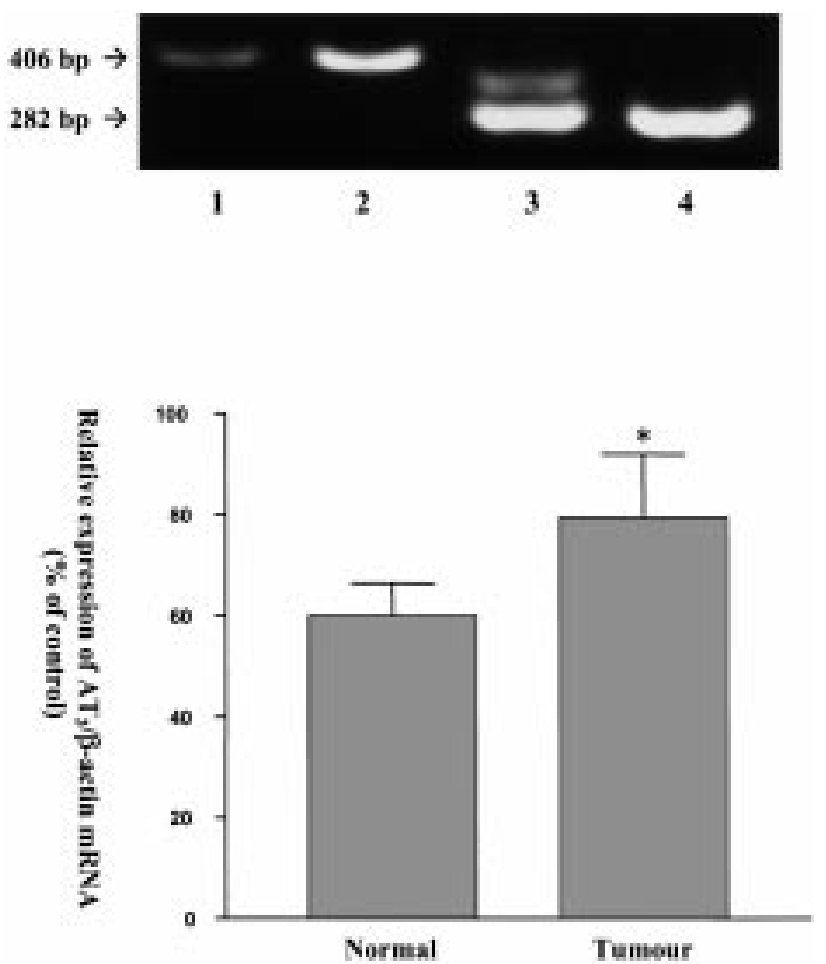

Figure 3 RT-PCR analysis of the mRNA expression for $\mathrm{AT}_{2}$ receptor in PETs. Lane 1, $\mathrm{AT}_{2}$ receptor expression in normal pancreas; lane 2, $\mathrm{AT}_{2}$ receptor expression in a PET; lane $3, \beta$-actin expression in the normal pancreas; lane $4, \beta$-actin expression in a PET. The arrows indicate the expected size of amplified products from $\mathrm{AT}_{2}$ receptor (406 bp) and $\beta$-actin (282 bp). The relative expression is indicated by $\mathrm{AT}_{2}$ receptor/ $\beta$-actin $\mathrm{mRNA}(\%$ of control) in normal and in PET pancreas. Data are means \pm S.E.M. ( $n=5$ PETs/group). * $P<0.05$.

\section{Localization of RAS components in normal pancreas and PETs}

In normal pancreas, immunoreactivity for angiotensinogen appeared to be localized predominantly in the pancreatic islets (Fig. 4A). Focal immunostaining for angiotensinogen was also found in the cytoplasm of the epithelial cells from pancreatic ductal epithelium (Fig. 4C). For $\mathrm{AT}_{1}$ receptor protein, the expression was localized to the pancreatic ducts (Fig. 4B and D). In PETs, angiotensinogen and $\mathrm{AT}_{1}$ receptor proteins were not detected in pancreatic islets (Fig. 4C and D). The specificity of the immunostaining for angiotensinogen and $\mathrm{AT}_{1}$ receptor was validated by the negative signal when the specific antibodies were preadsorbed with an excess of their respective peptide antigen (data not shown).

\section{Discussion}

There has been a steady stream of studies of the potential role of a local RAS in the non-human pancreas. The target for the pancreatic RAS could provide an insight into future management of several pancreatic diseases including acute pancreatitis, diabetes mellitus, cystic fibrosis and pancreatic cancer (19). Nevertheless, there is a lack of solid evidence for the presence of a local RAS in the human pancreas. The only piece of evidence available in the English language literature demonstrated the presence of $\mathrm{AT}_{1}$ receptor in human pancreas by means of immunohistochemistry (11). In the present investigation, we examined the different components of the RAS in the pancreas by immunohistochemistry and semi-quantitative RT-PCR. The mRNAs of angiotensinogen and angiotensin II receptors $\left(\mathrm{AT}_{1}\right.$ and $\left.\mathrm{AT}_{2}\right)$ were observed in human pancreas. Angiotensinogen protein was found predominantly in pancreatic islet and $\mathrm{AT}_{1}$ receptor was in the pancreatic ducts. Thus, an intrinsic RAS exists in the human pancreas.

Insulinoma is the most common type of PET. Unfortunately, no human insulinoma cell line is available for experimental analysis at the moment. A rat insulinoma cell line has been employed for the analysis of the properties of the pancreatic islet and PETs (20). The expression of genes encoding multiple peptide hormones including insulin, glucagon and somatostatin normally noted in the pancreatic islets, has been demonstrated in the rat insulinoma cell line (20). Moreover, the expression of angiotensinogen gene was found in rat insulinoma cell lines (21). The expression was regulated by glucocorticoid and thyroid hormone (22). This piece of evidence suggests that, at least in an animal model, an RAS may be involved in the pathogenesis of PETs. In the current study, we demonstrated, for the first time, an increase of angiotensinogen mRNA in insulinomas from human pancreas. Multi-hormone production in human PETs has been demonstrated by immunohistochemistry in our laboratories (12). It is tempting to relate the multi-potentiality of neoplastic islet cells to the developmental pathway of differentiation of islet cells. They are probably derived from a common stem cell of endodermal origin. The demonstration of multi-hormone production allows the detection of the diverse secretory potential of PETs. In the previous experiment, our immunohistochemical investigation showed evidence for multihormone production in $18 \%$ of PETs (12). In this study, PETs were shown to co-express insulin and angiotensinogen. The percentage for the detection of multi-hormones would probably rise if the number of hormones being tested were increased. It is plausible to suggest that the potential for protein complex and clinical manifestation should be noted in PETs.

In this study, the expression of receptors for angiotensin II was altered in patients with insulinoma. $\mathrm{AT}_{1}$ receptor mRNA was found to be down-regulated in PETs and $\mathrm{AT}_{1}$ receptor protein was not detected in PETs by immunohistochemistry. In contrast, mRNAs of angiotensinogen and $\mathrm{AT}_{2}$ receptor were up-regulated in PETs. However, we could not rule out the possibility 

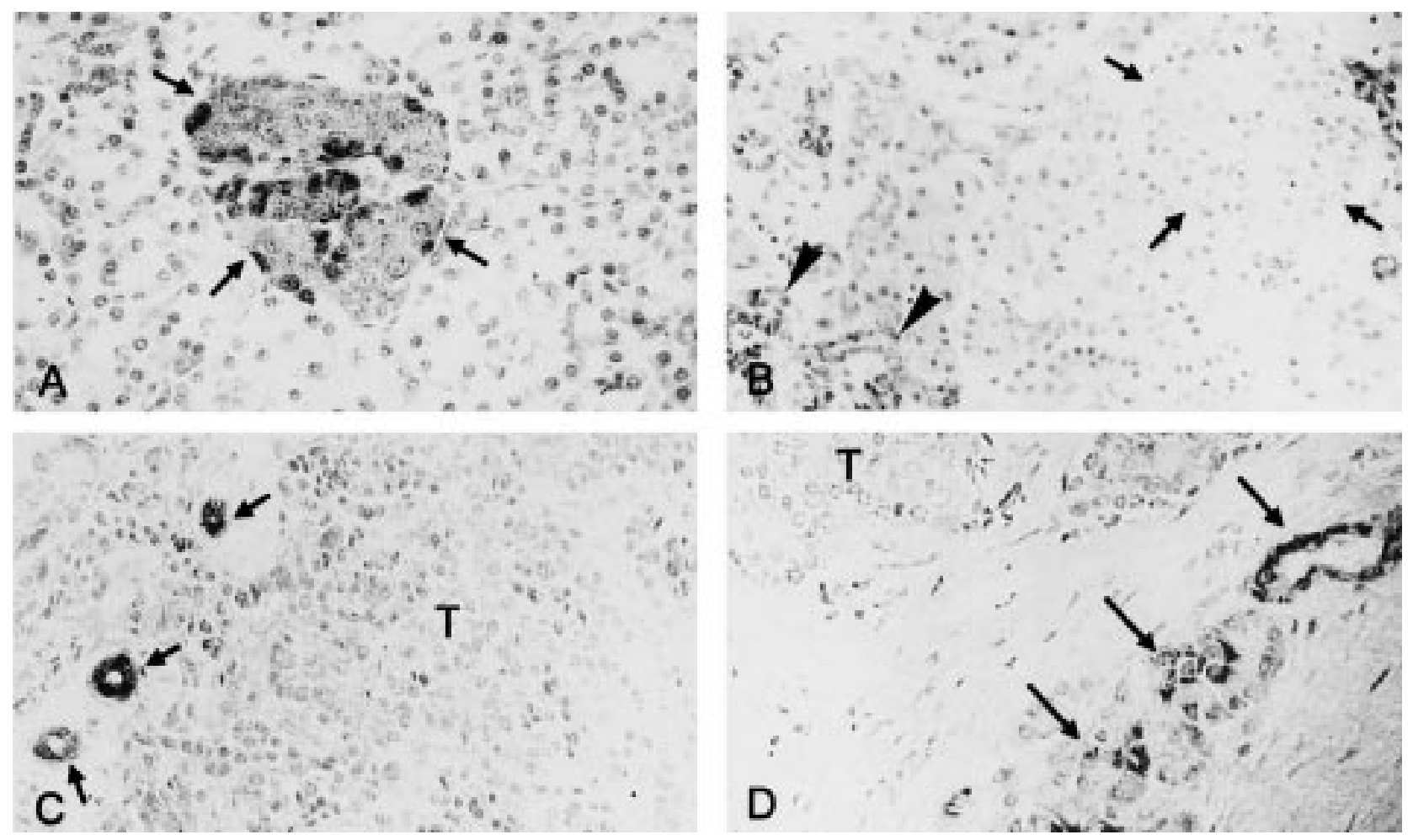

Figure 4 (A) Angiotensinogen expression in normal pancreas. Intense immunostaining is confined to the pancreatic islets (arrows). (B) $\mathrm{AT}_{1}$ receptor expression in normal pancreas. The immunostaining was noted in the pancreatic ducts (arrow heads). The islets (arrows) are negative for immunostaining. (C) Angiotensinogen expression in a PET. The tumour (T) is negative for immunostaining whereas the entrapped pancreatic ducts are positive for angiotensinogen (arrows). (D) AT 1 receptor expression in a PET. The tumour (T) is negative for $\mathrm{AT}_{1}$ receptor whereas the pancreatic ducts adjacent to the tumour shows a positive signal (arrows). Magnification $\times 60$.

that an increase of angiotensinogen was due to the aberrant expression by the pancreatic ducts with no expression in the endocrine neoplastic tissue. Previous studies have shown that pancreas is one of the few tissues that contains a significant proportion of the $\mathrm{AT}_{2}$ receptor as compared with that of $\mathrm{AT}_{1}$ receptor (2325). In view of this fact, it is likely that angiotensin II, increased via the up-regulation of its precursor angiotensinogen, could act on $\mathrm{AT}_{2}$ receptors in patients with PETs. A role for the up-regulation of the RAS in patients with PETs has yet to be determined. Blood flow to the pancreatic islets is regulated through a complex interaction of hormonal and nervous influences. The resultant islet blood perfusion controls the metabolism of the endocrine cells and the release of the islet hormones. One of the characteristics of PETs is their prominent vascular component. Angiotensin II has its principal action on the blood vessels. Interestingly, the association between pancreatic RAS and pancreatic blood flow has been recently reviewed in our laboratory (10). The angiotensin II produced in the PET may regulate the blood flow and hence stimulating islet hormonal secretion in the tumour.

It is worth noting that we demonstrated an increase in angiotensinogen mRNA whereas the angiotensinogen protein could not be detected in PETs. Two reasons may be postulated for this discrepancy: (i) PETs may contain abnormal angiotensinogen protein that could not be detected by the immunohistochemical approach, and (ii) the angiotensinogen protein may be carried away by the prominent vascular supply in the tumour at a high rate such that its low level in the tumour cannot be detected. Additional experimental data are required to fully elucidate the pathophysiological aspect of the action of the pancreatic RAS in PETs.

In summary, the present study provides solid evidence for the presence of a local RAS in the human pancreas. In addition, the different components of the RAS were subject to change in PETs. These changes may have physiological and pathophysiological relevance in PETs.

\section{Acknowledgements}

The authors would like to gratefully acknowledge support by Competitive Earmarked Research Grants from the Research Grants Councils of Hong Kong (Project No: CUHK 4116/01M and CUHK 4075/00M) and by Direct Grants from the Chinese University of Hong Kong. 


\section{References}

1 Page IH \& Bumpus FM. Angiotensins. New York: Springer Verlag, 1974.

2 Reid IA, Morris BJ \& Ganong WF. The renin-angiotensin system. Annual Review of Physiology 197840 377-410.

3 Peach MJ. Renin-angiotensin system: biochemistry and mechanisms of action. Physiological Reviews 197757 313-370.

4 Menard J, Clauser E, Bouhnik \& Corvol P. Angiotensinogen: biochemical aspects. In The Renin Angiotensin System, pp 8.1-8.10. Eds JIS Robertson \& MS Nichollas. London: Gower Medical Publishing, 1993.

5 De Gasparo M, Catt KJ, Inagami T, Wright JW \& Unger T. The angiotensin II receptors. Pharmacological Reviews $2000 \mathbf{5 2}$ $415-472$.

6 Campbell DJ. Circulating and tissue angiotensin systems. Journal of Clinical Investigation 198779 1-6.

7 Leung PS, Chan WP, Wong TP \& Sernia C. Expression and localization of the renin-angiotensin system in the rat pancreas. Journal of Endocrinology 1999160 13-19.

8 Leung PS, Chan HC \& Wong PYD. Immunohistochemical localization of angiotensin II in the mouse pancreas. Histochemical Journal $19983021-25$.

9 Chappell MC, Millsted A, Diz DI, Brosnihan KB \& Ferrario CM. Evidence for an intrinsic angiotensin system in the canine pancreas. Journal of Hypertension $19919751-759$

10 Leung PS \& Carlsson PO. Tissue renin-angiotensin system: its expression, localization, regulation and potential role in the pancreas. Journal of Molecular Endocrinology 200126 155-164.

11 Tahmasebi M, Puddefoot JR, Inwang ER \& Vinson GP. The tissue renin-angiotensin system in human pancreas. Journal of Endocrinology $1999161317-322$.

12 Lam KY \& Lo CY. Pancreatic endocrine tumour: 22-year clinicopathological experience with morphological, immunohistochemical observation and a review of the literature. European Journal of Surgical Oncology 199723 36-42.

13 Lam KY, Lo CY, Fan ST \& Luk JM. Telomerase activity in pancreatic endocrine tumours: a potential marker for malignancy. Molecular Pathology 200053 133-136.

14 Chomczynski P \& Sacchi N. Single-step method of RNA isolation by acid guanidinium thiocyanate-phenol-chloroform extraction. Analytical Biochemistry 1987162 156-159.
15 Chan WP, Fung ML, Nobiling R \& Leung PS. Activation of local renin-angiotensin system by chronic hypoxia. Molecular and Cellular Endocrinology $2000160107-114$.

16 Leung PS, Lam SY \& Fung ML. Chronic hypoxia upregulates the expression and function of $\mathrm{AT}_{1}$ receptor in rat carotid body. Journal of Endocrinology 2000167 517-524.

17 Leung PS, Tsai SJ, Wallukat G, Leung TN \& Leung TK. The upregulation of angiotensin II receptor $\mathrm{AT}_{1}$ in human preeclamptic placenta. Molecular and Cellular Endocrinology $2001 \mathbf{1 8 4}$ 95-102.

18 Leung PS, Chan WP \& Nobiling R. Regulated expression of pancreatic renin-angiotensin system in experimental pancreatitis. Molecular and Cellular Endocrinology 2000166 121-128.

19 A round table on renin-angiotensin system in the pancreas: from the basic research to the bedside, pp 1-57. In Journal of the Pancreas 2, Ed. PS Leung. Genoa: ES Burioni Ricerche Bibliografiche, 2001.

20 Philippe J, Chick WL \& Habener JF. Multipotential phenotypic expression of genes encoding peptide hormones in rat insulinoma cell lines. Journal of Clinical Investigation 198779 351-358.

21 Brasier AR, Philippe J, Campbell DJ \& Habener JF. Novel expression of the angiotensinogen gene in a rat pancreatic islet cell line: transcriptional regulation by glucocorticoids. Journal of Biological Chemistry 1986261 16148-16154.

22 Brasier AR, Philippe J, Campbell DJ \& Habener JF. Glucocorticoid and thyroid hormone regulation of angiotensinogen gene expression in a pancreatic islet cell line. Transactions of the Association of American Physicians 198699 13-19.

23 Chappell MC, Diz DI \& Jacobsen DW. Pharmacological characterization of angiotensin II binding sites in the canine pancreas. Peptides 199213 313-318.

24 Chappell MC, Jacobsen DW \& Tallant EA. Characterization of angiotensin II receptor subtypes in pancreatic acinar AR42J cells. Peptides $199516741-747$.

25 Chappell MC, Bosch SM, Hansen BC, Ferrario CM \& Diz DI. Differential expression of $\mathrm{AT}_{2}$ angiotensin II receptors in the primate pancreas. Journal of Hypertension 199412 S181.

Received 12 October 2001

Accepted 9 January 2002 